Bisera Dakova*

\title{
„Die Welt außerhalb“ in der Anthologie Auf der Insel der Seligen (1910) von Penčo Slavejkov
}

https://doi.org/10.1515/slaw-2019-0005

Abstract: The article scrutinizes the inimitable literary project On the Isle of the Blessed (Na Ostrova na Blažennite, 1910) by Pencho Slaveykov (1866-1912) from an innovative perspective: the inscription of the utopian island into extra-literary reality, taking into account the explicit geographical and cultural topoi used in the book. This analysis investigates relations of closeness / distance between those spheres in the work that are evidently fictional, and those that comment on real geographical spaces. It illuminates their reciprocal referencing and overlapping, in which fiction exists side by side with reality. In doing so, a number of the book's specific mental maps are reconstructed and related to the cultural meaning ascribed to "the Italian," "the French," "the Germano-Scandinavic," and not least the so-called "Own" ["nashensko"]. Taken together, these form the backbone of the value system of this geo-poetical literary project.

Keywords: Pencho Slaveykov, Bulgarian Modernism, fiction and extra-literary reality, cultural dichotomies of modernity, cosmopolitanism, settledness

\section{Einführung. Die Insel der Seligen als dritter Raum zwischen Fakt und Fiktion (oder: wie die Empirie die Fiktion umkodiert)}

Das originellste Werk des bulgarischen Modernisten Penčo P. Slavejkov Auf der Insel der Seligen (Na Ostrova na Blažennite, 1910), das seinen dichterischen Werdegang krönt, ruft immer wieder produktive Bezeichnungen hervor: „mystifizierte Anthologie“ (M. Nedelčev 1986: 5), „eine eigenartige Utopie der Literatur, ein Buch,

*Kontaktperson: Dr. Bisera Dakova, Universität Wien, Institut für Slawistik, Spitalgasse 2, Hof 3, Wien, Österreich und Institut für Literatur der Bulgarischen Akademie der Wissenschaften BAN, Šipčenski prochod 52, bl. 17, 1113, Sofia, Bulgarien, E-Mail: 2bisserka.dakova@univie.ac.at 
welches das grundlegende Gattungsbewusstsein des Kreises ,Misǎl' widerspiegelt, aber auch modifiziert“ (G. Tichanov 1998: 145), ,eine Insel-Bibliothek“(V.Stefanov 2000: 180), ,ein moderner Roman“(Sv. Igov 2006: 153), „eine Über-Anthologie“(N. Dimitrov 2012: 29), „das Borgesianischste bulgarische Buch“ (B. Kurtaševa 2012: 133), „ein wahres Panorama selbststigmatisierender Bilder“ (H. Schmidt 2013: 51) usw. ${ }^{1}$ Bei allen diesen radikalen Definitionsversuchen fällt auf, dass sie den eigentlichen Kern, das Undefinierbare dieses Werks, umkreisen. So als ob die frühe Feststellung aus dem Jahr 1927 (Pundev 1927: 375), es handele sich bei der Insel um eine „komplizierte, noch nicht endgültig erhellte Mystifikation“ “„,сложна, все още неизяснена мистификация"], noch gültig sei und nur immer weiter detailliert werden kann.

Unter den potenziell endlosen Definitionen, die sich aus der ambivalenten Gattungs- und Erscheinungsform des Buchs (Insel-Utopie, Insel-Metapher, Mystifikation, Kombination autobiographischer und intertextueller Bezüge etc.) ergeben, gehört - meiner Meinung nach - die treffendste Michail Nedelčev, der von einer „mystifizierten Anthologie“ spricht. Dabei muss man natürlich berücksichtigen, dass das so bezeichnete Projekt zwei spekulative, ,unechte‘ Ausprägungen der referenzierten Gattungen miteinander verknüpft: Es handelt sich schließlich um eine Anthologie einer fremden, imaginierten, nicht-existenten Literatur und gleichzeitig um eine Mystifikation, die auf dem Realen/Tatsächlichen/Empirischen beruht. Diese Benennung/Beobachtung korreliert mit der These von G. Tichanov (1998: 146), dass in Slavejkovs Buch zwei Gattungsmodelle (Anthologie und Mystifikation) reaktualisiert werden. Diese werden dabei jedoch nur anzitiert, innovativ transformiert, aber nicht den Erwartungen des Lesers entsprechend verwirklicht. Jeder wissenschaftliche Interpret dieses hybriden Werks muss sich im Prozess der Analyse dennoch eines konkreten Begriffs bedienen, um seine Deutung durchführen zu können (Dimitrov 2012: 37). ${ }^{2}$

Das Thema der folgenden Betrachtungen ergibt sich aus dem Umstand, dass die Anthologie Penčo Slavejkovs in ihrer spielerischen Fiktion keine hermetische ,Insel' konstruiert und abspaltet, sondern im Gegenteil, dass in diesem seltsamen Buch ein kommunikativer Raum aus komplizierten Bezügen zur ,Realität‘ erschlos-

1 Im bulgarischen Original entsprechend: „мистифицирана антология“; „своеобразна утопия за литература, като книга, която отразява, но и модифицира основни идеи от жанровото съзнание на кръга ,Мисъл““; „остров-библиотека“; „модерен роман“; „свръхантология“; „най-борхесовската българска книга“.

2 Nikola Dimitrov z.B. hat ausführliche Definitionen des Buchs formuliert, um es letztendlich in seiner Abhandlung doch nur als „Anthologie“ zu benennen. 
sen wird. ${ }^{3}$ Durch diese scheinbar sich selbst verleugnende Mystifikation wird der Status sowohl des Fiktionalen (der im Buch porträtierten Insel-,Wirklichkeit‘) als auch des Realen (der im Buch explizit erwähnten historischen und biographischen Fakten) ambivalent. Beide bestimmen sich gegenseitig. Das, was wir im Buch als ,Realität‘ erkennen (Daten, Fakten, Ereignisse, Personen), dient dazu, das Fiktionale/Imaginäre (die erfundenen Dichter-Persönlichkeiten, den kulturellen Status der Insel-Literatur) näher zu erklären und zu deuten. Oder, genauer gesagt, zu übertragen. Schließlich positioniert sich der Verfasser der Anthologie als ihr Übersetzer, als Transponierender einer fremden Poesie. Mich wird der Status der Fiktion im Folgenden dahingehend interessieren, inwieweit diese aus verschiedenen Realitätskomponenten zusammengesetzt ist und von diesen getragen wird. ${ }^{4}$

Aus diesem Grund lasse ich die zahlreichen impliziten Anspielungen und indirekten intertextuellen oder kulturellen Verweise in der Anthologie außer Acht (immer wieder geben diese Anlass zu spannenden Spekulationen), sondern konzentriere mich auf die hier so genannte ,Welt außerhalb،, also auf das, was als ,tatsächlich‘, ,empirisch', ,wirklich geschehen' oder als real existent gilt. Anders formuliert stellt sich der Artikel die folgenden Fragen: Wie funktioniert die ,Realität‘ im Buch, wozu wird sie genutzt, wird sie der ,Insel-Wirklichkeit‘ untergeordnet oder - ganz im Gegenteil - ihr übergeordnet? Wie durchdringen sich in dieser Mystifikation, die sich als solche selbst offenbart, ,Fakt' und ,Fiktion'? Oder werden wir vom „Effekt der Realität“ (Kurtaševa 2012: 135-136), die verstreut unter den fiktionalen Tatsachen und Topoi wahrzunehmen ist, affiziert? Die folgenden Beobachtungen zielen auf eine Rehabilitierung der verdrängten Geographie des Buchs, die bis dato oft überlagert wurde durch die Dominanz des Bibliographie-Narrativs (der zahlreichen erfundenen Bücher, die auf der gleichfalls erfundenen Insel existieren), wenn wir einer geistreichen Feststellung Biljana Kurtaševas (2012: 135) zustimmen wollen.

Gerade dank dieser äußerst ,verwickelten‘ Verhältnisse, so die These, bildet sich in Slavejkovs Buch-Projekt etwas Drittes aus - die ,Insel-Realität‘, die überzeugend und plausibel wirkt. Dabei handelt es sich um einen entlegenen Raum der Marginalität und der ,undurchsichtigen', intransparenten Öffentlichkeit.

3 Hier trete ich in einen gewissen Widerspruch zu der These von Galin Tichanov, dass Slavejkovs Insel Ausdruck „eines freiwillig gewählten räumlichen Isolationismus“ [„един доброволно избран пространствен изолационизъм“] gewesen sei (Tichanov 1998: 159).

4 Diese Auseinandersetzung mit dem Buch Auf der Insel der Seligen schlägt einen konträren Weg ein zur Analyse von Henrike Schmidt „Anthologiefiktion. Penčo Slavejkovs Auf der Insel der Seligen und seine postmoderne Rezeption“ (2013), wo der Akzent auf dem Fiktiven und seiner Bedeutung für die Kanon-Bildung liegt. So wäre die Untersuchung der Realität im Buch gleichzeitig ein Beitrag zur paradoxalen Erforschung der Fiktion. 
Penčo Slavejkov als fiktiver Herausgeber der Anthologie charakterisiert diese Insel-Welt als „verwildert“ (in der Skizze über den Dichter Spiro Godina etwa wird sie „Divogonska eparchija“ genannt, d.h. „eine das Wilde jagende Eparchie“; Slavejkov 1939: 37), und das trotz aller die Gesellschaft erschütternder politischer Umwandlungen, die gleichzeitig beschrieben werden, trotz all der eifrigen Nachahmungen der modernsten Erscheinungen in der Literatur, der Philosophie und angrenzenden kulturellen Bereichen.

\section{Vermittlungsprozesse zwischen inner- und außerliterarischer Welt}

Die „Insel der Seligen“ ist eine Welt der gebrochenen Widerspiegelungen, eine sekundäre Welt, die übersetzt und gedeutet werden muss, die eine Erklärung, eine kulturelle Transponierung und eine Sichtbarkeit braucht. Deshalb wird die ,Welt außerhalb، als ein Vehikel der Deutung - der Gleichsetzung und der Abgrenzung - angeführt und angewandt. Man könnte die These vertreten, dass Penčo Slavejkovs Vision der Insel der Seligen im Ganzen auf den Effekten der Abgrenzung/Entfremdung von und der Annäherung an die ,Welt außerhalb“ beruht. Das Erscheinungsbild dieses imaginären und dabei doch glaubwürdigen Zeit-Raum-Kontinuums wird durch diese zwei entgegengesetzten Dynamiken konstruiert.

Dieses Prinzip - der Entfernung/Entfremdung einerseits und der Annäherung andererseits - wird bereits im Vorwort eingeführt, wenn der Pseudo-Herausgeber seine unglückliche Erfahrung mit der ins Englische übersetzten Volksliedsammlung The Shade of the Balkans (1904, London) ${ }^{5}$ thematisiert. Der fiktive Verfasser kontrastiert hier nämlich die „gute“ und die „missglückte“ Öffentlichkeit: In England hat die Anthologie die ihr zustehende Anerkennung gefunden, „bei uns“ aber „hat kein Hahn danach gekräht“ beziehungsweise, in der Idiomatik des Originals, „sich niemand aus irgendeinem Winkel“ dazu gemeldet [„никой от никое кюше не се обади“] (Slavejkov 1939: 5). So wird bereits direkt am Anfang die grundlegende Opposition zwischen England (bzw. Europa oder allgemein dem Raum der Offenheit und der Öffentlichkeit) und „bei uns“ (oder dem Raum des Unsrigen - „našensko“) aufgerufen. Durch diese Opposition, die im Buch

5 Biljana Kurtaševa nennt The Shade of the Balkans Slavejkovs ,vergessene“ Anthologie und charakterisiert sie als „невъзможната антология“ („die unmögliche Anthologie“; 2012: 90108). 
unaufhörlich variiert wird, sich vertieft und entwickelt, wird die ,Welt außerhalb“ konstruiert. Gleichzeitig ergibt sich hieraus eine dramatisch gespaltene Weltwahrnehmung, gespalten zwischen der ersehnten kulturellen Wirklichkeit einerseits und „našensko“ (dem Unsrigen) andererseits, letzteres offensichtlich zurückgeblieben, konservativ, deformiert. Viele sonst unerklärliche Phänomene der Insel, wie die Existenz der Universität in Anvart, die Büchergesellschaft oder das Café der Armenier, können aus dieser dramatischen Spannung heraus erhellt werden. Das ,Unsrige‘ oder ,bei uns‘, diese Termini konnotieren einen vertrauten Umgang mit der erfundenen Insel-Wirklichkeit. So erscheinen viele Phänomene dieser fiktionalen Welt als selbstverständlich, keiner weiteren Erklärung bedürftig. Die Verweise auf das ,Unsrige - direkt oder implizit - sind zahlreich. Sie funktionieren zuverlässig als Mittel der Analogie. Aber nicht nur die Analogie wird in der Anthologie gesucht und hervorgehoben: Während dem fiktiven Dichter Vidul Fingar ein Gedicht zugeschrieben wird, dessen Titel einen bulgarischen Topos bezeichnet („Na Šipka“ / „Auf dem Šipka-Pass“, Slavejkov 1939: 21), um seine Dichtung durch ,unsrige‘, vertraute Realien zu konkretisieren, wird in der Skizze über Stamen Rosita ganz anders verfahren. Rosita hat selbst im sogenannten ,Unsrigen“ gelebt, hat also direkte Berührung mit diesem sonderbaren Territorium, hat sich davon - obwohl meist negativ - inspirieren lassen und das Buch „Auf dem Balkan“ („Na Balkana“) geschrieben (Slavejkov 1939: 96). Das entlegene ,Unsrige“ wird also zum poetischen Objekt eines fiktiven Dichters, wodurch diese scheinbar ausgedachte und trotzdem plausible Projektion gleichsam belebt wird. Resultat ist das seltsame Zusammentreffen Stamen Rositas, eines erklärten Nietzsche-Apologeten, als Vertreter der Insel und gleichzeitig als Repräsentant der ,Welt außerhalb“ mit der groben geistigen Realität des ,Unsrigen“. Oder, anders formuliert: die allegorische Berührung einer fiktionalen Figur mit der Realität, mit der tatsächlichen, familiär-vertrauten Wirklichkeit.

Oft ist aber auch die umgekehrte Tendenz zu beobachten: Angesehene Dichter der Insel treten in direkte Verbindung mit der ,Welt außerhalb', d.h. mit dem ,Nicht-Unsrigen', mit bedeutungsvollen weltbekannten Realien. Dabei geht es keinesfalls nur um einen flüchtigen Kontakt: Diese Dichter, nur formell der Insel angehörig, ${ }^{6}$ verweilen, ,leben“ an ganz anderen Orten in Europa, wo sie gleichfalls nicht ansässig sind, wo sie auch nicht hingehören, an Orten, die sie zu Ausgangspunkten ihrer unaufhörlichen Reisen machen. Die Wanderungen des Dichters Ticho Čubra z.B. erweisen sich als emblematisch - von Paris aus begibt er sich nach Spanien, Schweden, Norwegen usw. Der Pseudo-Herausgeber der Anthologie gibt vor, ihn in Nürnberg kennengelernt zu haben - ein guter Grund,

6 Galin Tichanov (1998: 168) spricht von den „Immigranten“ der Insel. 
den Aufenthaltsort und die räumliche Fixierung des Anthologisten selbst in Frage zu stellen. Die Position ,zwischen den Räumen und Orten“ kennzeichnet auch die Figur des Verfassers.

\section{Die kulturelle Geographie der Insel-Fiktion}

Eine solche dynamische Existenz ist auch für den ersten der fiktiven Dichter in der Anthologie charakteristisch, für den revolutionär gesinnten Anhänger Giuseppe Garibaldis mit Namen Bore Vichor (Slavejkov 1939: 10-11). Seine Biographie weist eine Reihe merkwürdiger ,Fakten` auf: Vichor kämpft und stirbt in Italien für die Sache einer fremden Nation; er stammt von der Insel, hat aber nie dort gelebt. Seine Persönlichkeit und sein dichterisches Talent entfalten sich fern von ihr sein ganzes Leben und Werk drücken einen ungestillten, aufrührerischen Geist aus, der nur formell die Heimat-Insel vertritt.

Auf einer abstrakteren Ebene ist es interessant $\mathrm{zu}$ betrachten, wie das ,Italienische' im Buch im Allgemeinen fungiert, d.h. wie Slavejkov als Verfasser und vorgeblicher Anthologist mit dieser geographischen und kulturellen Realität umgeht. ,Italien“ und das ,Italienische“ wirken hier mehrdimensional: Zunächst einmal ist ,Italien“ der ersehnte Raum dramatischer politischer Kollisionen und der bereits erkämpften nationalen Identität. Seine Positionierung als Kulturnation hingegen ist ambivalenter: Einerseits erweist sich das ,Italienische“ als ein unerreichbares Kulturmuster. Andererseits wird es im Buch konsequent profaniert, beispielsweise durch die misslungenen Nachahmungen und Plagiate italienischer Dichter seitens des Insel-Poeten Velko Meruda (Slavejkov 1939: 46). Die hohen Werte der italienischen Dichtkunst werden durch eine komische, quasi herzzerreißende Dichtung ersetzt und mit dieser gleichsam ,verwechselt" (in diesem Sinne ist die Gleichsetzung von Zitaten der real existierenden Poeten italienischer Herkunft mit ihren fiktiven, wortwörtlichen - und durchaus jämmerlichen - Kopien besonders wirkungsvoll, Slavejkov 1939: 46).

An dieser Stelle muss angemerkt werden, dass die biographische Skizze über den Dichter Velko Meruda eine Kontaminierung der beiden literarhistorisch existenten Personen Ivan Vazov (1850-1921) und Konstantin Veličkov (1855-1907) und somit eine deutlich polemische Auseinandersetzung mit diesen beiden etablierten Schriftstellern der bulgarischen Literaturgeschichte darstellt. ${ }^{7}$ Wir wissen, wir ver-

7 Von der ursprünglich geplanten Anspielung (auf Dimităr Veliksin, 1840-1896), dokumentiert in einer vom Autor hinterlassenen Liste mit den Namen der fiktiven Dichter und ihren jeweiligen Prototypen, hat sich Slavejkov beträchtlich entfernt. Nur das Thema der geistigen Umnachtung und des Lebensendes bleibt erhalten. 
muten, wir sind davon überzeugt, dass die Person K. Veličkov in diesem Text alludiert wird - durch die reiche Palette von Anspielungen auf Ereignisse aus seinem Leben (sein Aufenthalt in Florenz, seine Gattungsvorliebe für Sonette, die Tätigkeit als Minister, als Redakteur einer bekannten Zeitschrift und Literaturkritiker). Sein Name aber taucht in der Skizze und überhaupt im Buch konkret nie auf. Er ist von der Insel-Wirklichkeit verschlungen und assimiliert worden, ist in einen Bestandteil dieser Fiktion verwandelt worden. Er gehört zu der Insel, obwohl er nicht ein einziges Mal persönlich genannt wird. Mit seinen markanten Zügen und Gesten aber war er für den damaligen bulgarischen Leser deutlich erkennbar. (Vielleicht ist Slavejkovs Buch in diesem Sinne ein Markstein in der bulgarischen Literaturgeschichte bezüglich der Positionierung Veličkovs - die Anthologie erlaubt es diesem nur, im mächtigen Schatten Ivan Vazovs sichtbar zu werden, lediglich als eine Komponente des Vazovschen Schaffens betrachtet zu werden -, als so unauflöslich sollte sich die ,Verwachsenheit' der beiden Autorfiguren erweisen.)

Was ist aber das Charakteristische an Veličkov, das in der biographischen Skizze über Velko Meruda so heftig parodiert und verhöhnt wird? Welche seiner literarischen Verdienste bleiben verdunkelt und ignoriert? Zunächst sollen seine besondere Bindung an die französische Literatur sowie seine Übersetzertätigkeit erwähnt werden. Letzteres hat die schriftstellerische Entwicklung Veličkovs besonders geprägt. Während seines gesamten Schaffens ist er vorwiegend als Übersetzer und Anthologist tätig. Die meisten Übersetzungen der wirkmächtigen und für den künftigen Kanon konstitutiven Bulgarischen Chrestomathie (Bălgarska Christomatija, 1884) stammen von ihm. D.h. beide Rollen - des Übersetzers und des Anthologisten -, mit denen Penčo Slavejkov in seinen Anthologien so frei umgeht, sind in einem bestimmten Maß Veličkov eigen. Außerdem hat sich der Literat realiter am Aprilaufstand des Jahres 1876 beteiligt, wird danach eingekerkert und gibt seine Erlebnisse in der Haft aus einer inneren, psychologischen Erzählperspektive wieder. Seine Memoiren V tămnica (Im Kerker) aus dem Jahr 1899, in denen auch seine emblematischen „Zarigrader Sonette“ („Carigradski soneti“) veröffentlicht wurden, stellen einen neuen Entwicklungsschritt in dieser Gattung dar.

In allen seinen Aktivitäten (nicht nur als Politiker und Minister) zeigt Veličkov ein starkes Engagement, eine bestimmte Einfühlung in die Sache - besonders betrifft dies seine Auseinandersetzung mit dem ,Italienischen'. Er ist ein vorzüglicher Kenner der italienischen bildenden Künste. Sein epistolarer Text Briefe aus Rom (Pisma ot Rim) ist das (anfechtbare) Zeugnis dieser Kompetenz. Penčo Slavejkov erblickt hier ausschließlich Kompilation und Plagiat. Der Essay ist aber auch der innovative Reisebericht eines feinfühligen Gebildeten. Veličkov übertrug Dantes Inferno ins Bulgarische - eine unübertroffene Übertragung bis heute. Außerdem übersetzte er weitere italienische Dichter in die Heimatsprache, darun- 
ter eben jenen Lorenzo Stecchetti, der in Slavejkovs Skizze über den fiktiven Meruda ausdrückliche Erwähnung findet. Veličkov identifizierte sich mit dem Italienischen, indem er unter anderen auch ein italienisches Pseudonym - Jovanni Beatrafio - benutzte.

Wollte man sein literarisches Gesamtwerk auf einen emblematischen Nenner bringen, so wäre dies das Sonett, worauf es in der Biographie Velko Merudas eine aufdringliche Anspielung gibt. Als Literaturkritiker zeichnet er sich durch seine Nachsicht, seine Großzügigkeit und eine enorme Begeisterung für junge Literaturtalente aus - eine Eigenschaft, die von Slavejkov verlacht wird. In seiner Zeitschrift Letopisi (1899-1905) publiziert er Gedichte der noch unbekannten, später etablierten und kanonisierten Autoren Teodor Trajanov und Dimităr Bojadžiev. Sein ganzes Leben ist gespalten zwischen der intensiven Teilnahme am politischgesellschaftlichen Leben in Bulgarien und der ,erhabenen' Verbannung in der Fremde (Florenz, Konstantinopel, Grenoble). Im Unterschied zu der örtlich stabilen Figur Ivan Vazov ist er keine sesshafte Autor-Persona.

Der Klassiker Ivan Vazov im Gegenteil wird in der imaginären Dichter-Biographie Velko Merudas direkt erwähnt und wohlwollend-familiär besprochen als quasi naturgegebenes Element des hiesigen Raums, des bereits vielfach genannten ,Unsrigen'. Im komplizierten System des Buches wird er im Weiteren dennoch durchaus ambivalent figurieren. Sein Autorenname repräsentiert die ,wirkliche“ Wirklichkeit (Slavejkov 1939: 124). Seine emblematischen Dichtungen aber - der Marsch „Stille, weiße Donau“ (,Tich bjal Dunav“), der Roman Unter dem Joch (Pod igoto), dessen berühmte Szene „Die Tafel bei Čorbadži Marko“ („Trapezata u čorbadži Marko“) bewusst fälschlich als Titel desselben ausgegeben ist - werden mit Absicht nur beiläufig erwähnt und als anspruchsvolle, aber in ihrer Profanität berüchtigte Betitelungen karikiert: ${ }^{8}$ „С културата и нейните завоевателни задачи тоя театър е толкова роднина, колкото Вазовата Трапеза у чорбаджи Маркосо Симпозиона на Платона“ [„Mit der Kultur und ihrer expansiven Mission ist dieses Theater in dem Maße verwandt, wie Vazovs Tafel bei Čorbadži Marko mit Platons Symposion."]” .

8 Verweise auf Ivan Vazov kommen in unterschiedlichen biographischen Skizzen vor. In den Texten über Vidul Fingar und Njagul Kavela wird dieser vom Verfasser der Anthologie anonymisiert und absichtlich trivialisiert (wenn man literarische Mittelmäßigkeit als beständige Profanierung der Literatur versteht, so wird das Werk Vazovs hier auf eine Ebene mit den populärsten Texten der damaligen Zeit gestellt). In der Skizze über Ivo Dolja hebt der Verfasser hervor, dass das erste Kapitel des berühmten Romans Pod igoto (Unter dem Joch) keinen philosophischen Kern besitze (Slavejkov 1939: 22, 124, 206).

9 Der tatsächliche Titel des ersten Kapitels im Roman lautet „Gost“ („Gast“). Sinn der Änderung ist vermutlich eine so absichtlich gesuchte wie absurde Analogie zwischen der „Tafel“ (dem reich gedeckten Tisch) und dem Gastmahl bei Platon. 
Auf diese Art und Weise - durch die kontaminierte Gestalt von Velko Meruda in ihrem Oszillieren zwischen zwei zentralen Personen der bulgarischen Literaturgeschichte sowie zwischen dem ,Unsrigen' und der ,Welt außerhalb“ - wird das italienische Sujet im Buch Slavejkovs aktiviert. Es erreicht hier einen Höhepunkt, um später als eine erhabene, bereits validierte Präsenz des Ausländischen (nicht aber des Fremden) wieder auf den Plan zu treten. In der Skizze über die Dichterin Silva Mara wird das ,Italienische‘ dann auch schon wie nebenbei spontan erwähnt. Erstaunlicherweise hat die unter Pseudonym tätige Dichterin (vom Verfasser lediglich „Schreibende“ genannt) nämlich eine italienische Übersetzerin, mit dem wohlklingenden Namen Pagalini. Hier scheint in der Fiktion eine für die damalige Zeit realiter phantastische Situation auf: Das anonyme dichterische Werk einer Verfasserin, die aus einem marginalen Kulturraum stammt und sich hinter Pseudonymen verbirgt, wird in drei Sprachen übertragen! Die italienische Übersetzerin Pagalini ist nur das konkrete Beispiel für dieses Wunder der Übersetzung.

Das ,Italienische“ nistet in den verschiedensten Schichten der Anthologie, auch in dem auf den ersten Blick parodistischen Namen der fiktiven Stadt Ranava (in der Skizze über Spiro Godina, vgl. Slavejkov 1939: 36), der auf die reale italienische Stadt Ravenna anspielt. Und schließlich wird die Insel Capri prominent erwähnt, in der Biographie über den Dichter Njagul Kavela, als dessen Refugium. Sie verweist gleichzeitig auf Maksim Gor'kij und den bulgarischen Schriftsteller Petko Todorov, die sich beide dort niedergelassen hatten, auf zwei Autorenfiguren also, die unterschwellig präsent sind, ohne sichtbar auf der Textoberfläche $z u$ erscheinen. Nicht zuletzt bezeichnet der Name Capri auch eine Insel, diesmal aber eine existente: eine Insel voller Idylle und Frieden, die wahre Insel der Seligen, die sich im Diesseits befindet. Resümierend lässt sich zusammenfassen: In Penčo Slavejkovs Buch wird die Insel der Seligen einerseits räumlich abgesondert, andererseits vollzieht sich auch der gegenteilige Prozess. Diesem engen und in keiner Weise autarken Raum werden verschiedene andere Räume entgegengesetzt und im Gegenüber entfaltet. So existiert die Insel in der Sphäre des Empirischen durchaus ebenbürtig unter den vielfältigen geographischen Topoi. In der für den Autor typischen Ironie figuriert sie dabei nicht zuletzt als die unwirtliche Alternative zu einer idyllischen Insel wie Capri. Die detailliert imaginierte Insel-Fiktion tritt in wirkliche räumliche Verhältnisse ein und repräsentiert damit eine ,echte' Lebensmöglichkeit.

Während ,Italien“/das ,Italienische“ einen deutlichen Bezug zum gesegneten Land herstellt, existieren in der Anthologie andere Räume und Orte, die über einen noch höheren Grad an Ambivalenz verfügen. Da wäre zum Beispiel die ausdrücklich negativ konnotierte Zone der Krankheit, der „Hypernervosität“, der Verrücktheit, der geistigen Umnachtung, allgemein: der schmerzlichen Welt- 
erfahrung. Diese wird Paris als der Metropole der Nervosität und der steten Unruhe zugeschrieben (vielsagend in dieser Hinsicht wäre die gestrichene, in das Buch nicht aufgenommene biographische Skizze über Bone Vojdan ${ }^{10}$ ). Andererseits begeben sich aufrührerische Geister wie Bojko Razdjala nach Paris, um an diesem Ort - vergebens - Heilung für ihre zerrütteten Seelen zu suchen. In jedem Fall aber wirkt Paris als Anziehungstopos, als Raum des echten, vollwertigen Lebens, nämlich in den Skizzen über die Dichter Bojko Razdjala, Sekul Skăta, Ticho Čubra. Darüber hinaus entwirft der Verfasser ergänzend zur etablierten Semantik des Südlichen aber auch das ,skandinavisch-deutsche“ Areal als einen Gegenpol zum ,Italienischen'.

Wenn wir über solche, durchaus amorphen Räume in Slavejkovs Anthologie sprechen, dürfen diese nicht buchstäblich verstanden werden. Es handelt sich vielmehr um Konstellationen kultureller Auswirkungen und Einflüsse, um Nachklänge, aber auch um eigenartige geistige Transformationen. An vorderster Stelle sind die in weltanschaulichen Widersprüchen lebenden Anhänger Friedrich Nietzsches oder Max Stirners zu nennen, wie in der Skizze über Kitan Dožd mit Nachdruck hervorgehoben wird. So entsteht und entwickelt sich im Buch (sowie im gesamten Werk) das Meta-Thema der persönlichen Prägung Slavejkovs durch Friedrich Nietzsche, vor allem durch dessen Konzept des Übermenschen und seine unendlich variierenden Anklänge und Deutungen. Nicht zufällig wird im pseudobibliographischen Apparat der Insel erwähnt, dass das Poem „Hymnen auf den Tod des Übermenschen“ („Chimni za smǎrtta na svrăchčoveka“) in luxuriösem Druck und reich illustriert herausgegeben worden ist. Zudem trägt sein Autor den vielsagenden bulgarischen Namen „Razdjala“ („Abschied“, „Trennung“; Slavejkov 1939: 150). Die Gattung Hymne bezeichnet hier ihrerseits einen rituell-festlichen Abschied von den innigst erlebten Bildern und Ideen. Die emblematische Autorschaft steht im bewussten Gegensatz zu den vielen Insel-Poeten in der Anthologie, die anonym oder durch ein Pseudonym eingeführt werden, zu den Autoren, die umstrittene, in ihrem Status zweifelhafte Dichtungen geschaffen haben (Manuskript gebliebene oder nie veröffentliche Ausgaben), wie z.B. Spiro Godina und Vitan Gabăr. Innerhalb der Anthologie lässt sich also eine präzise Differenzierung zwischen zwei Autoren-Typen konstatieren: Einerseits wird die Insel von Dichtern mit prekärer Autorschaft bewohnt (z.B. Bore Vichor, Nedan

10 Der fiktive Dichter Bone Vojdan ist ein ausgewiesener Gegner des Französischen und in dieser Hinsicht ein Vertreter des Deutschen: „Той се е учил в Париж, но е французомразец, жесток и непримирим, като да се е раждал в Берлин“ [„Er hat in Paris studiert, aber er ist ein Franzosenhasser, grausam und unversöhnlich, so als ob er in Berlin geboren wäre“] (Slavejkov 1958: 343). In dieser Skizze wird auch Maksim Gor'kij explizit erwähnt und die Anspielungen auf Petko J. Todorov sind noch prägnanter. 
Vetko, Vitan Gabăr, Vita Deleda), auch sind Namen wie Velko Meruda und Ticho Čubra keine echten Namen, sondern Pseudonyme, d.h. Quasinamen. Andererseits erscheinen im Buch eine Reihe von Literaten, die durch ihre Autorennamen und deren emblematische Werktitel überzeugend wirken. Diese starke Differenzierung ist durch den jeweiligen kulturellen Raum bedingt: Die Dichter, deren Autorschaft mehr oder weniger unsicher ist, befinden sich tatsächlich auf der Insel und sind der dort herrschenden Marginalität ausgesetzt. Die Figur Bore Vichor ist eine signifikante Ausnahme, aber bei dieser Figur dominiert das Sagenhafte (die Heldentat) und deswegen erscheint ihre Autorschaft als nebensächlich. Die anderen, die namhaften und einflussreichen Dichter in der Anthologie leben ausschließlich andernorts, so als ob ihre Berühmtheit a priori von einem nichtmarginalen Ort ausgeht und sich ausschließlich ihrer extrem mobilen Lebensweise verdankt.

\section{Kosmopoliten und Sesshafte. Räumliche und kulturelle Dichotomien. Schlussfolgerungen}

So stößt man auf eine grundlegende (wie ich glaube, bis jetzt unbemerkt gebliebene) Opposition in der Anthologie Slavejkovs zwischen zwei Arten von Dichterfiguren, idealtypisch repräsentiert in den folgenden fiktiven Autoren: Erstens Ticho Čubra, der unermüdliche Reisende, der Eroberer neuer Räume und fremder Weltanschauungen, und zweitens Neno Večer, der Epikureer, der die geistige Freiheit besingt (es handelt sich hier um eine offensichtliche Anspielung auf den literaturgeschichtlich verbürgten Autor Kiril Christov), dabei aber vergnüglich in der Heimat verweilt und das Große im Kleinen, im Vertrauten, „in den schwarzen Augen seiner Geliebten“ entdeckt. Für den damaligen (wie auch den heutigen) bulgarischen Leser, der die Andeutung auf den hedonistischen Wanderer Kiril Christov leicht entziffert, wirkt die idyllische Existenz von Neno Večer in der Heimat - diese ausgesprochene Gleichgültigkeit gegenüber der großen Welt eher als eine Kuriosität.

Diese beiden Dichtertypen der Slavejkovschen Anthologie sind die zwei unbenannten Pole der Insel, die wie alle Gestalten in diesem Werk auch aporetisch wirken: der stürmisch Erlebende, der Unruhige, der alle möglichen Räume durchquert, und der sesshafte Genießer des Lebens. Dazwischen können wir Sekul Skăta einordnen, wegen seiner eher akademischen Gebundenheit an Orte wie Paris und London und wegen seiner im Text kompliziert ausgedrückten Abneigung gegen das Städtchen Lourdes - ein Topos der religiösen Obsession. Ähnlich wie Ticho Čubra gehört Sekul Skăta dezidiert nicht zur Insel, zu diesem begrenzten 
und bedrückenden Raum. Er ist ein Weltbürger, ein Kosmopolit. In diesem Sinne markiert er die finale Position in der Reihe der Kosmopoliten, die im Buch genannt werden und denen eine Serie von konkret räumlich verorteten Dichtern folgt: Njagul Kavela, Ralin Stubel, Nedan Vetko, Vitan Gabăr, Vita Deleda. Es handelt sich bei letzteren immer um Autor`innen, deren künstlerische Bestrebungen lediglich mehr oder weniger gelungene Nachahmung sein können und die deswegen unausweichlich in Vergessenheit oder in eine ,missglückte‘ weil dysfunktionale Öffentlichkeit geraten, kulturelle Eigengewächse also, die nicht unter den Nenner einer konventionellen Literaturklassifikation gebracht werden können (so eigensinnig-autochthon ist z.B. Vitan Gabăr, der Verfasser von Epitaphen, ein ungebildeter Mann, dem jede schriftstellerische Eitelkeit fremd ist; Slavejkov 1939: 289). Die Anthologie Slavejkovs endet mit dem biographischen und künstlerischen Porträt einer Frau, deren Existenz wiederum als zweifelhaft (ins Sagenhafte führend) dargestellt wird. Vita Morena verfolgt ihre erhabenen Ideale nicht in „spitzen Schuhen auf hohen Absätzen“ [„, теснички обущта с високи токове“], sondern bahnt sich ihren Weg in den „Sandalen des Lebens“ [„коравите сандали на живота“] (Slavejkov 1939: 255) - mit diesem unkonventionellen Bild schafft Slavejkov einen Ausdruck für eine sonderbare Form der Pilgerschaft, die bewusst auf eine unkonventionelle Außenseiterposition abzielt.

An dieser Stelle soll daran erinnert werden, dass in der folgenden Anthologie von Penčo Slavejkov, Deutsche Dichter (Nemski poeti) aus dem Jahr 1911, das ,Weibliche“ oder, anders gesagt, der weibliche Blickwinkel erneut eingesetzt wird, um das Gesamtbild der porträtierten deutschen Poesie zu relativieren. D.h. das ,Weibliche“ wird in dieser zweiten, nicht weniger seltsamen Anthologie unerwartet aufgewertet. Die Bewertungen gegenüber der deutschen Poesie als solcher, die im Brief der „deutschen Dichterin“ [„,немската поетка“] offenbar werden, positionieren sich gegenüber der Dichtung der Männer nachlässig resümierend. Man verspürt eine gewisse Herablassung, z.B. in den folgenden metaphorischen Bildern, durch welche die männliche Dichtung charakterisiert wird: „мъглата на техните стихове“ [„der Nebel ihrer Verse“]; „и чиито чувства са винаги причесани, от гребена на традицията“ [„,deren Gefühle immer frisiert sind vom Kamm der Tradition“] (Slavejkov 1911: 168).

Was nun die Gesamtschau des Buchs Auf der Insel der Seligen angeht, so setzt sich zum Ende hin, wie wohl nicht anders zu erwarten, ganz gesetzmäßig das Bild ihrer insularen Abgelegenheit durch. Auf diese Weise wird die Problematik des Insel-Daseins vollendet und abgerundet. Was aber nicht bedeuten soll, dass die anthologische Mystifikation Penčo Slavejkovs eine Lösung, einen Ausweg zeigt aus den Dilemmata kultureller Dichotomien und Ungleichgewichte - einfach deswegen, weil die Anthologie eines solchen Autors kein Buch der Lösungen und der Auswege sein kann. 


\section{Literaturverzeichnis}

Dimitrov, Nikolaj. 2012. Antologično-panteonni implikacii na kanona. In Plamen Antov, Marineli Dimitrova \& Georgi Gospodinov (red.), Antologii i antologijno - meždu avtora i teksta, 2742. Sofija: Institut za literatura BAN.

Igov, Svetlozar. 2006. Kniga za Penčo Slavejkov. Varna: Slavena.

Kurtaševa, Biljana. 2012. „Sjankata na Balkana“ - (ne)vǎzmožnata antologija; Podstǎpi kǎm Na Ostrova na blaženite. In Dies., Antologii i kanon: antologijni modeli na bǎlgarskata literatura, 33-138. Sofija: Prosveta.

Nedelčev, Michail. 1986. Zavrǎštane kăm „Na Ostrova na blaženite“. In Penčo Slavejkov, Na Ostrova na blažennite, 3-15. Sofija: Bălgarski pisatel.

Pundev, Vasil. 1927. Dvete antologii na Penčo Slavejkov. In Zlatorog VIII, H. IX. 375-386.

Schmidt, Henrike. 2013. Anthologiefiktion. Penčo Slavejkovs Aufder Insel der Seligen und seine postmoderne Rezeption. In Bisera Dakova, Henrike Schmidt, Galin Tihanov \& Ludger Udolph (Hrsgg.), Die bulgarische Literatur der Moderne im europäischen Kontext. Zwischen Emanzipation und Selbststigmatisierung, 47-74. München, Berlin \& Washington: Sagner.

Slavejkov, Penčo P. 1911. Nemski poeti. Sofija: Al. Paskalev.

Slavejkov, Penčo P. 1939. Na Ostrova na blažennite. Sofija: Chemus.

Slavejkov, Penčo P. 1958. Săbrani săčinenija v osem toma. T. 2. Sofija: Bălgarski pisatel.

Stefanov, Valeri. 2000. Učastta Vavilon. Lica, maski i dvojnici v bălgarskata literatura. Sofija: Anubis.

Tihanov, Galin. 1998. Žanrovoto săznanie na krăga „Misăl“. Kăm kulturnata biografija na bălgarskija modernizăm. Sofija: Akademija.

\section{Anhang}

Literaturgeschichtliche Persönlichkeiten, die in der Anthologie Erwähnung finden. Die Liste folgt ihrem Erscheinen im Buch.

Henry Bernard / Berlyne; Hector Berlioz; Giuseppe Garibaldi; Theodor Storm; Lorenzo Stecchetti; Enrico Panzacchi; Téophile Gautier; Hippolyte Taine; Jens Peter Jacobsen; Max Stirner; Henry Thoreau; Knut Hamsun; Franz Grillparzer; Sappho; Alkäus; Catull; Reinhold Niebuhr; Johann Wolfgang von Goethe; Heinrich Heine; Friedrich Nietzsche; Tacitus; Oscar Wilde; Platon; William Gladstone; Henrik Ibsen; Bjørnstjerne Bjørnson; Erwin Rohde; Francis Jammes, Franziskus von Assisi; Saint-Paul-Roux; Henry George; Auguste Rodin, Lev Tolstoj; Ivan Turgenev; Michail Lermontov; Ralph Waldo Emerson.

Nicht explizit erwähnte literaturgeschichtliche Persönlichkeiten, die in der Anthologie vermutlich angedeutet werden. Die Liste folgt ihrem Erscheinen im Buch. 
Christo Botev; Petko Račev Slavejkov; Ivan Vazov; Konstantin Veličkov; Pejo K. Javorov; Petko J. Todorov; Maksim Gor'kij; Kiril Christov; Stojan Michajlovski; Gustav Falke; Richard Dehmel; Detlev von Liliencron; Carlo Pazachani.

Im Buch genannte Toponyme (nach ihrem Erscheinen im Buch geordnet).

Chios; Rom; Sizilien; Wien; Paris; Balkan; Spanien; Fjorde; Schweden; Norwegen; Nürnberg, Piräus; Lourdes; Capri. 